\title{
A risk control framework for safe manufacturing workstations in view of COVID-19
}

\author{
Ebly Sanchez $z^{1, *}$ and Knut Åkesson ${ }^{2}$ \\ ${ }^{1}$ Volvo Group Truck Operations, Greensboro, USA \\ ${ }^{2}$ Department of Electrical Engineering, Chalmers University of Technology, Sweden
}

\begin{abstract}
The manufacturing industry resumes operations after the COVID-19 pandemic supported by return-to-work guidelines, which are mostly personal protection measures for the workers and employees. In this paper, we propose a framework for assessing risk at the workstation level by linking the risk levels to possible mitigation strategies that can be implemented using standard operating procedures (SOP), $5 \mathrm{~S}$ and problem-solving. Within industrial plants, operators work in close contact with coworkers and supervisors, and they are also sharing tools and machines. It is therefore, essential to develop strategies that reduce the operator's exposure to viruses in the workplace. The purpose of this work is that when implemented, the risk assessment model and specifically how SOP, 5S and problem solving can be used to implement administrative and engineering controls resulting in a safe workplace and increasing level of confidence for the operators working within the plant.
\end{abstract}

\section{Introduction}

Many uncertainties remain concerning when the COVID19 coronavirus will reach its peak in every country and globally. At present, the therapeutic strategies to deal with the infection are only supportive, and prevention aimed at reducing transmission in the community is our best weapon [2]. It is clear that COVID-19 has disrupted operations on a massive scale, challenging manufacturers to assess the impact continually and, in the best way, respond quickly and preventively. The manufacturing industry is resuming operations supported by return-to-work guidelines [3-5,9], which is providing the needed virus protection for workers and employees. Because COVID-19 cannot be eliminated and is likely to be around for a long time, it is highly necessary to increase the safety and trust of the workers and employees working at manufacturing sites. Therefore, managers need guidance on how to motivate the trust of the workers and at the same time secure the daily production process with the safety of the employees at the highest priority. Resuming operations while developing and implementing COVID-19 prevention measures has been not only a challenge but also a massive learning process where new knowledge is being developed regarding more proactive steps to avoid virus transmission in the future.

The risk of disease transmission of COVID-19 depends on several factors. Some of these factors are described in the U.S. Department of Labor and U.S. Department of and Health and Human Services' [2] which include:

- Distance between workers. Manufacturing workers often work close to one another on production or assembly lines. Workers may also be near one another at other times, such as when clocking in or out, during breaks, or in locker/changing rooms.

- Duration of contact. Manufacturing workers often have prolonged closeness to coworkers (e.g., for 8-12 hours per shift). Continued contact with potentially infectious individuals increases the risk of COVID-19 transmission.
- Type of contact. Manufacturing workers may be exposed to the infectious virus through respiratory droplets in the air - for example, when workers in a plant have the virus cough or sneeze. It is also possible that exposure could occur from contact with contaminated surfaces or objects, such as tools, workstations, or break room tables. Shared spaces such as break rooms, locker rooms, and entrances/exits to the facility may contribute to their risk.

The main contribution in this paper is a framework that combines existing approaches to risk assessment, risk control approaches, standard operating procedures to implement risk control, 5S, and problem-solving for a sustained improvement cycle. We provide an example of how to the risk assessment together with the evaluation of different risk control approaches to reduce risk levels. Also, an example of how a standard operating procedure can be used to implement an administrative risk control approach at the workstation level is presented.

The outline of the paper is that the different techniques are briefly introduced in section 2 . The proposed model is presented in Section 3. In Section 4 it is discussed how the proposed model can be continuously updated and reviewed.

\section{Background}

In this section, we give an introduction to the hierarchy of controls that categorize possible means to handle hazards.

\subsection{Risk analysis}

Risk analysis, [1], is routinely used in the manufacturing industry to assess the risk for physical accidents at the workstation. However, the risk analysis in the manufacturing industry has not traditionally included potential exposure to viruses. The risk priority number (RPN), which is the product of the severity, occurrence, and detection, 


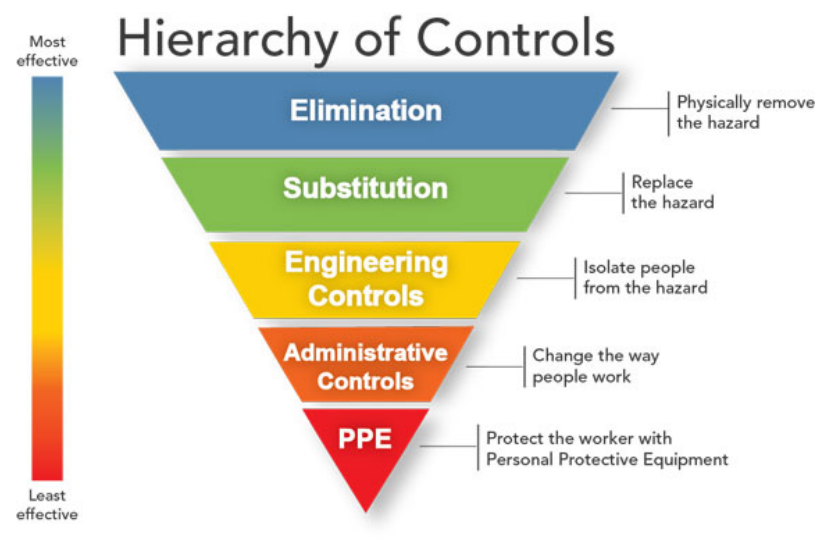

Fig. 1. Hierarchy of Controls (HOC), from [10].

is used to prioritize potential risks against each other to focus on those risks that have the most considerable consequences. In this work, we introduce a valid and straightforward approach to risk analysis similar to the RPN approach but adapted to handle risk related to exposure to the virus.

\subsection{Hierarchy of controls (HOC)}

Controlling exposures to occupational hazards is the fundamental method of protecting workers. Traditionally, a hierarchy of controls, see Fig. 1, has been used as a means of determining how to implement feasible and effective control solutions. Risk controls were categorized using a modified version of the National Institute for Occupational Safety and Health (NIOSH) hierarchy of risk controls [10].

Elimination Stop using the hazardous process or materials.

Substitution Substitute a less hazardous process or material.

Engineering controls With a focus on physical barriers, isolation, forcing functions, human factors, and failsafe design, engineering controls improve safety independent of worker interactions.

Administrative controls Policies, procedures, training, and other controls that depend on people taking the correct actions.

Personal protective equipment (PPE) such as facemasks, face-shields, safety glasses, and gloves.

Elimination and substitution are the most effective approaches, while PPE is typically considered the least effective approach. In this work, we mainly focus on what can be done at the engineering control and the administrative controls level to reduce risk at the workstation.

\subsection{Standard Operating Procedures (SOP)}

SOP [7] has become the base for improvement in the industry with clear benefits. In the SOPs, it is precisely described how to perform the job, i.e., it describes the step sequence. The use of SOPs typically leads to a reduction of variation and error correction, improved safety, facili- tates communication, and increasing labor discipline. In this work, we assume the SOP has the following characteristics.

- Simplicity and visualization, the worker immediately easily found and understood the necessary instructions.

- OK and not OK (NOK) conditions. An easy way for the operators to visually understand what is $\mathrm{OK}$ and NOK when performing their jobs.

- The possibility of rapid changes in process parameters,

- Clarity, which ensures that every worker has all relevant activities in the process as well.

- The ability to monitor the implementation of SOP and its impact on the process parameters.

When all workers at the workstations perform their tasks consistently according to SOPs, less quality, delivery, and safety concerns happen in the production line. In this paper, we discuss how SOP can be used to implement administrative controls in HOC.

\section{$2.45 \mathrm{~S}$}

5S [6] stands for five Japanese words: Seiri (sort), Seiton (set in order), Seiso (shine), Seiketsu (standardize), and Shitsuke (sustain), is a system where waste is reduced, safety is improved, and quality are optimized through observing an orderly work area. The 5S defines five steps. The first step (Seiri) sort eliminates unnecessary, broken items from the work area. The second step (Seiton) set in order focuses on providing efficient storage areas for the remaining items. Shine, the third step, is to clean the work area thoroughly. Daily schedules for cleaning the area are created to sustain these changes. Once the first three steps have been implemented, the next step is to standardize the best practices in the work area. In this work, we propose that SOP are created or enforced if already available. The newly developed practices are integrated into the SOP, and they become the standard way of performing actions.

\subsection{Problem solving}

Manufacturing or safety problems need to be described consistently and systematically to allow for a common understanding and avoid recurrence of same problems. The following steps are a common methodology applied today in industry for problem-solving [8]:

1. Team formation

2. Problem description

3. Interim containment action

4. Root cause analysis

5. Corrective actions

6. Validate corrective actions

7. Implement corrective actions a standardize (update SOP)

8. Team recognition

Problem-solving in our risk assessment model supports continuous improvement and learning loop. During the SOP observations and dialogue between the workers and team leaders, risks and gaps are identified and docu- 
mented. The problem-solving process is used to learn and continuously improve the way of working at the workstations.

\section{Proposed model}

The proposed model consists of the the following parts.

a) Risk assessment

b) Risk control strategies

c) Continues improvement and learning with problem solving

The risk assessment model starts with the identification of the hazards. If we take the distance between workers as an example, the hazards would be "social distancing not possible with current workstation set up". In a typical car or truck assembly line, depending on the line-balancing situation and volume requirement, there could be more than two people working closely together, which would be a high-risk situation. To determine and prioritize the risk, a table with the exposure and probability criteria has been defined. See Table 1. The levels of exposure ' $E$ ' and probability ' $P$ ' should be defined by a cross-functional team of safety experts and operations. Once $E$ and $P$ have been decided, the results which are $E \cdot P$ is an indication of the level of the risk. The higher the value, the higher is the risk. The highest risk should be selected to develop the next steps to define the administration and engineering control actions.

The risk control strategies are based on HOC. While HOC contains five controls, we focus on the engineering controls and administrative control in this paper. Examples of engineering controls could be to isolate people from exposure to the virus by, for example, separating people from each other, this can be done by increasing the takt-time (line balancing) and thus need fewer operators at the workstation. Examples of administrative controls, can be disinfection of tools before and/or after a work shift, and how operators are expected to move within the workstation to guarantee minimum distance to other operators. Administrative controls can preferably be documented using SOPs to ensure that all operators (experienced and inexperienced) follow the same working standard. It is taken for granted that PPE is already widely in use at all manufacturing sites, which are providing most of the prevention measures today. The control actions, once defined, need to be included as OK and NOK conditions in the SOPs and in the $5 \mathrm{~S}$ standards to be followed by the team members as they perform the operations at the workstations.

For continuous improvement and learning, SOPs and $5 \mathrm{~S}$ should be the standard way of working at any manufacturing site to secure that employees adhere to safety and health regulations. Deviations from meeting SOP and requirements can result in errors leading to infection control. The following are some pre-requisites and methods for effective use of the SOPs:

- Clear roles and responsibilities of the supervisors, team leaders, and team members regarding the development, usage, and evaluation of SOPs.

- Current SOP's availability and visibility at locations
Table 1. Exposure and probabilities.

\begin{tabular}{|c|c|c|c|}
\hline Exposure $(E)$ & & Probability $(P)$ & \\
\hline \hline Low & 1 & Improbable & 1 \\
Medium & 2 & Remote & 2 \\
High & 3 & Occasional & 3 \\
Very high & 4 & Probable & 4 \\
& & Frequent & 5 \\
\hline
\end{tabular}

close to the work areas.

- Clear scheduling for SOP observation. It is recommended daily for team leaders and weekly for supervisors.

- Secure the team members can perform the job according to the SOP by providing dedicated on-thejob training.

- Confirm team members are following the SOP. A job observation between team leaders and the team members of the workstations should be conducted daily for effective SOP compliance.

In summary, the proposed framework are as follows:

a) Identification of the hazards.

b) Determine the risk according to the exposure ' $E$ ' and the probability ' $P$ ', according to Table 1 .

c) Determine the level of the risk by multiplying $E \cdot P$.

d) Prioritize the hazards according to the risk level. Follow the sequence below for hazards, starting with those that have the highest level of risk.

e) Determine if any administrative control or engineering control actions are possible.

f) Calculate updated risk levels for the control actions.

g) Define OK and NOK conditions and include in SOP and $5 \mathrm{~S}$.

h) Team members at workstations work according to SOP and $5 \mathrm{~S}$.

i) Continuously identify deviations from $\mathrm{SOP}$ and $5 \mathrm{~S}$.

j) Apply problem-solving to improve and resolve deviations.

k) Continue to reduce the level of the hazards.

Next, we will follow up with an example of how the proposed model can be used to evaluate different risk control strategies.

\subsection{Example}

In Table 2, we can describe HOC via an example, to illustrate more specifically the HOC risk assessment. Lets develop an example with the hazard described in the previous section, which is the social distance not possible with the current workstation setup. Before the type of HOC actions are developed, the risk need to be decided. The cross-functional team decided that this is a high risk of exposure to the virus transmission, which is level $3(E=3)$. The team also decided that the level of probability was frequent, which is level $5(P=5)$. Therefore the risk level in this example is $E \cdot P=15$. The first action decided by the team was to reduce the worker density per workstation, 
Table 2. Risk assessment model.

\begin{tabular}{|c|c|c|c|c|c|c|c|}
\hline Hazards & $E$ & $P$ & $E \cdot P$ & Action & new $E$ & new $P$ & new $E \cdot P$ \\
\hline $\begin{array}{l}\text { Social distancing not possible with } \\
\text { current workstation set up }\end{array}$ & 3 & 5 & 15 & $\begin{array}{l}\text { CA 1: Reduce the workers den- } \\
\text { sity per workstation, utilizing the } \\
\text { length of the chassis and the width } \\
\text { of the cab to meet social distanc- } \\
\text { ing requirements wherever possible } \\
\text { and max one operator per cab at } \\
\text { time. Use face mask all the time } \\
\text { and face shields where social dis- } \\
\text { tance requirement are not possible. }\end{array}$ & 1 & 5 & 5 \\
\hline $\begin{array}{l}\text { Social distance not possible with } \\
\text { current workstation set up }\end{array}$ & 3 & 5 & 15 & $\begin{array}{l}\text { CA 2: Comply with CA } 1 \text { and inte- } \\
\text { grate both the OK and NOK condi- } \\
\text { tions described in SOPs and follow } \\
5 \mathrm{~S} \text { standards. }\end{array}$ & 1 & 3 & 3 \\
\hline $\begin{array}{l}\text { Social distance not possible with } \\
\text { current workstation set up }\end{array}$ & 3 & 5 & 15 & $\begin{array}{l}\text { CA3: Comply with CA1, CA2 and } \\
\text { close gaps from SOPs and } 5 \mathrm{~S} \text { with } \\
\text { problem solving }\end{array}$ & 1 & 2 & 2 \\
\hline
\end{tabular}

utilizing the length of the chassis and the width of the cab to meet social distancing requirements wherever possible and max one operator per cab at a time. Use face-mask all the time and face shields where social distance requirements are not possible. The new exposure was reduce to $E=1$, which is a low exposure and the probability level stayed the same at $P=5$. Therefore the risk level became after the engineering control action $E \cdot P=5$, which indicates a reduction of 10 points in the risk level. The next administrative action taken by the team was to use SOPs and $5 \mathrm{~S}$ to comply with the first action. Examples of SOPs are shown on Fig. 2 and 3. To secure that the people at the workstation use the SOPs, the team decided to use daily SOP and $5 \mathrm{~S}$ observations. As a result of this new action, the exposure level stayed at level 1, which is low, however, the probability of the risk dropped from frequent to occasional, giving an overall risk improvement from 5 to 3. Finally, during the daily SOP and 5 S observations, the teams identified some deviations that could be improved to reduce the virus transmission further. These deviations and gaps were resolved by using problem-solving. After the application of problem-solving the exposure kept a low exposure level 1, however, the probability was further improved to remote, which is level 2. Therefore the new overall risk level became 2 .

The previous risk assessment example included a reference to SOP for implementing administrative controls. A similar example could be applied with an SOP for disinfection. Fig. 2 shows the general information about the SOP, while Fig. 3 shows the visual instructions.

\section{Continuous evaluation}

The main objective of the SOP and $5 \mathrm{~S}$ evaluation is to secure compliance to avoid virus transmission and to secure new SOPs are developed for new identified hazards like, maintenance work would be an issue, job rotation and line balancing. We recommend the following steps to evaluate

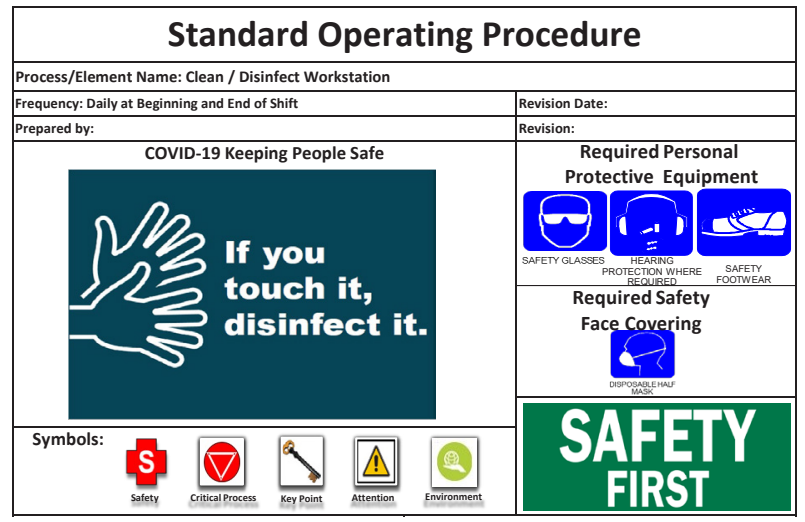

Fig. 2. SOP for disinfection. General information about the SOP.

the SOPs and $5 \mathrm{~S}$.

- Capture and document deviations and gaps from the job observations performed between team leaders/supervisors and workers at the workstations. These gaps should be classified according to what is controllable by the teams at the multi-workstations and what is not controllable.

- Visualize and prioritized these gaps so that issues can be resolved using problem-solving.

- Update risk and HOC actions and improve the SOPs based on the gaps that have been found and resolved.

- Visualize the number of gaps open/close, and the number of SOPs updated accordingly. These CI should be correlated to the plant's health and safety

\section{Qualitative evaluation}

The proposed approach has been evaluated using interviews with line managers at several part distribution centers in the USA. The risk assessment model provides a 


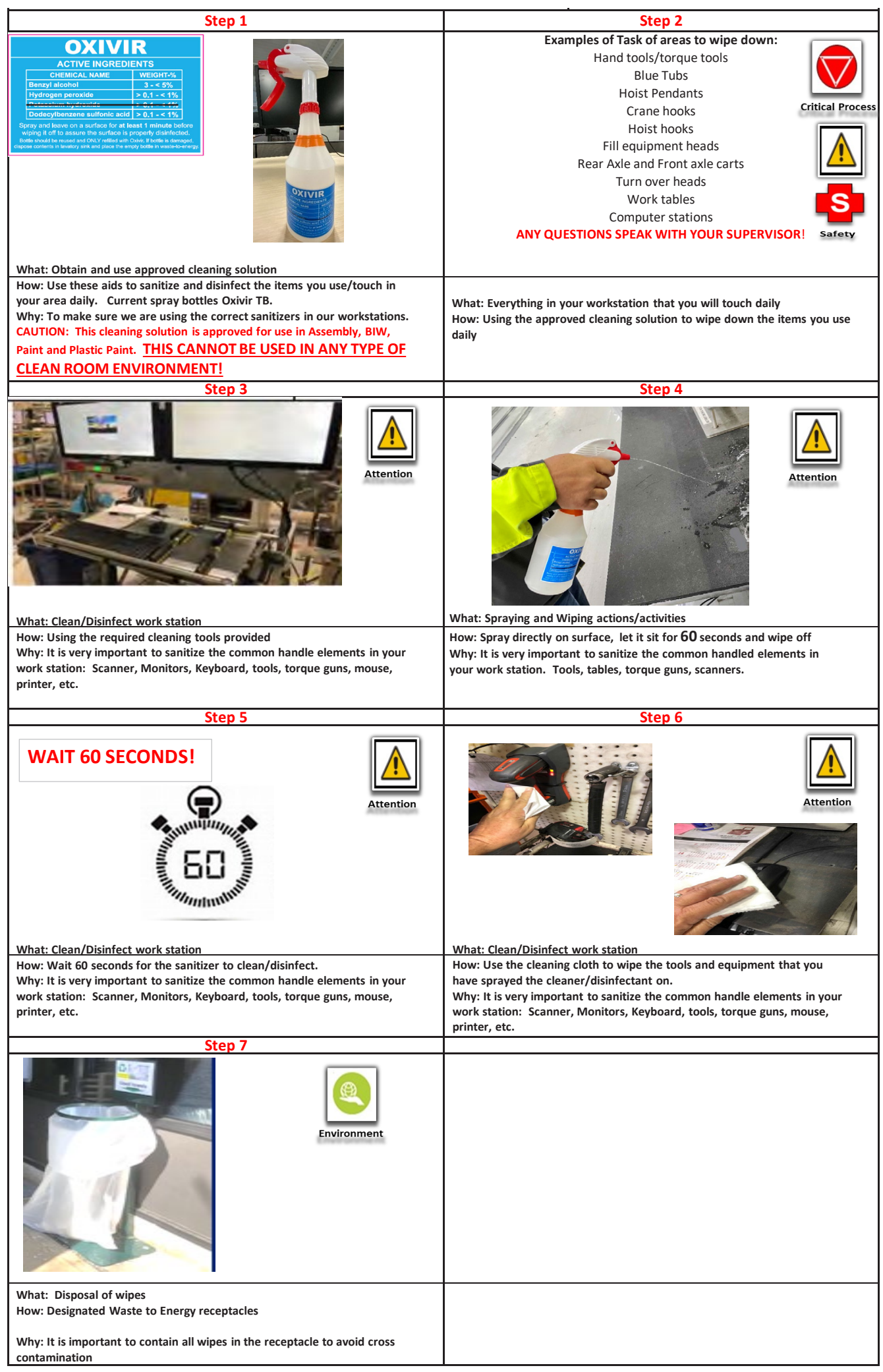

Fig. 3. SOP for disinfection. Detailed instructions for the individual steps. 
new way of pandemic prevention, but still at the administrative control level of the HOC pyramid. The SOP focuses on this qualitative evaluation because the SOP precisely described how to perform the job. It does not only helps the operators to understand the sequence of a specific operation. The OK and NOK conditions but also the SOP helps the first line manager to facilitate communication and increasing labor discipline when he conducts the SOP confirmation process.

In our interviews with line managers, we asked the following questions.

- Why do you conduct the SOP confirmation routine, and how COVID-19 is affecting it?

- What are the strength and weaknesses of this process?

We put these questions to several first-line managers in two distribution centers. Here is some feedback from them:

- Initially, I took for granted that the operators were following the SOP. If we have problems, then I come to discuss with the worker with the help of the SOP if he/she understands the job and if she/he is following the standard. This is the first thing I do to understand a possible problem. During this pandemic, we have been requested to do it at least one time per day, which has helped us a lot to improve the communication with the workers and to secure that the COVID-19 prevention measures are followed.

- I have learned a lot with the SOP confirmation process. I was doing it daily because it helped me to train new operators. During COVID-19 we have had a lot of changes of workers. The SOP confirmation process helped me to help the workers. Even when the operators understand the job, we followed the daily routines of SOP confirmation to understand any risk about COVID-19. The workers feel safer when we come and talk to them about COVID-19 risks.

- The SOP confirmation routine is helping me to generate ideas for improvement in my area. During COVID-19 we have some problems with the workers not following the prevention measures, especially the use of face mask. During the SOP confirmation process, I was able to identify why some workers did not want to use the face mask.

The main results of this SOP confirmation process have been the trust of the workers coming to work. Absenteeism has been high with more than 15 percent in some sites, but those sites that have been following the SOP confirmation process the absenteeism has been less than ten percent. As we prepare in the near future to increase production volume, this SOP confirmation will improve both the trust of the workers to be safe, and therefore the absenteeism would be lower.

\section{Conclusion}

Risk assessment and the corresponding improvement actions should become a highly prioritized process to increase the trust of the workers and employees. This paper presented a methodology based on risk assessment, stan- dard operation procedure, $5 \mathrm{~S}$, and problem-solving to help identify and evaluate possible actions to be taken to establish a safe workplace. The model is in the process of being implemented and evaluated in industrial plants. We expect the proposed model to be useful to reduce risk and to capture the new learning like the improvement of the rotation of the workers, increase the versatility of the workers, increase the priority of $5 \mathrm{~S}$ and SOP at every workstation, increase the frequency of the line balancing process, just to indicate a few examples.

\section{References}

1. T. Aven. Foundations of Risk Analysis. John Wiley \& Sons, 2012.

2. M. Cascella, M. Rajnik, A. Cuomo, S. C. Dulebohn, and R. D. Napoli. Features, evaluation and treatment coronavirus (COVID-19). StatPearls [Internet]. Treasure Island (FL): StatPearls Publishing; 2020 Jan-, 2020.

3. Fiat Chrysler Automobiles. Let's keep each other safe - return to work packet. Online; accessed 9-June2020, 2020.

4. Ford Motor Company. Return to work manufacturing playbook - working together to remain safe and healthy at work. Online; accessed 9-June-2020, 2020.

5. General Motors. Returning to the workplace with confidence. Online; accessed 9-June-2020, 2020.

6. H. Hirano. 5 Pillars of the Visual Workplace: The Sourcebook for $5 S$ Implementation. New York: Productivity Press, 1996.

7. M. Míkva, V. Prajová, B. Yakimovich, A. Korshunov, and I. Tyurin. Standardization - one of the tools of continuous improvement. Procedia Engineering, 149:329 - 332, 2016. International Conference on Manufacturing Engineering and Materials, ICMEM 2016, 6-10 June 2016, Nový Smokovec, Slovakia.

8. A. Smalley. Four Types of problems, from reactive troubleshooting to creative innovation. Lean Enterprise Institute, Cambridge, MA, USA, 2018.

9. Tesla. Return to work playbook. Online; accessed 9-June-2020, 2020.

10. The National Institute for Occupational Safety and Health (NIOSH). Hierarchy of controls. https://www.cdc.gov/niosh/topics / hierarchy/default.html, 2015. [Online; accessed 24-July-2020]. 